\title{
OPEN Effect of hybridized local and charge transfer molecules rotation in excited state on exciton utilization
}

\author{
Gang Sun ${ }^{1}$, Xin-Hui Wang ${ }^{1}$, Jing Li $^{1}$, Bo-Ting Yang ${ }^{1 \bowtie}$, Ying Gao ${ }^{2 \bowtie}$ \& Yun Geng ${ }^{3}$
}

The fluorescent molecules utilizing hybridized local and charge-transfer (HLCT) state as potential organic light-emitting diodes materials attract extensive attention due to their high exciton utilization. In this work, we have performed the density functional theory method on three HLCTstate molecules to investigate their excited-state potential energy surface (PES). The calculated results indicate the $T_{1}$ and $T_{2}$ energy gap is quite large, and the $T_{2}$ is very close to $S_{1}$ in the energy level. The large gap is beneficial for inhibiting the internal conversion between $\mathrm{T}_{1}$ and $\mathrm{T}_{2}$, and quite closed $S_{1}$ and $T_{2}$ energies are favor for activating the $T_{2} \rightarrow S_{1}$ reverse intersystem crossing path. However, considering the singlet excited-state PES by twisting the triphenylamine (TPA) or diphenylamine (PA) group, it can be found that the TPA or PA group almost has no influence on $T_{1}$ and $T_{2}$ energy levels. However, the plots of $S_{1}$ PES display two kinds of results that the $S_{1}$ emissive state is dominated by charge-transfer (CT) or HLCT state. The CT emission state formation would decrease the $\mathrm{S}_{1}$ energy level, enlarge the $S_{1}$ and $T_{2}$ gap, and impair the triplet exciton utilization. Therefore, understanding the relationship between the $S_{1}$ PES and molecular structures is important for designing highperformance luminescent materials utilizing HLCT state.

Organic light-emitting diode (OLED) was widely studied since the first small molecules device was reported by Tang et al. ${ }^{1}$. The luminescent materials, as the most important component of OLED, were always the focused study by materials scientists ${ }^{2-6}$. One most important requirement of the luminescent materials was a high photoluminescence quantum yield (PLQY). The pure organic materials utilizing hybridized local and charge-transfer (HLCT) state ${ }^{7-9}$ become potential high-efficiency OLED materials considering the low cost, less toxicity, and high exciton utilization.

Numerous highly luminous HLCT-state compounds containing triphenylamine (TPA, Fig. 1) group have been reported ${ }^{10-15}$. For example, in 2014, a series of twisting donor-acceptor (D-A) molecules with high exciton utilization efficiencies and full-color-range emissions were reported ${ }^{16}$. To enhance the proportion of radiative exciton, methods such as constructing orthogonal cyano substituted D-A structure imidazole derivative ${ }^{17}$, designing butterfly-shaped D-A chromophore ${ }^{18}$, modulating a fine proportion of charge-transfer (CT) state and locally excited (LE) state ${ }^{19}$ and choosing an appropriate substituted position, were suggested ${ }^{20}$. Especially, a pure-blue emitting molecule consisting of phenanthroimidazole and phenylcarbazole-substituted anthracene (PAC, Fig. 1) was reported, and the external quantum efficiency (EQE) exceeds $10 \%{ }^{21}$. Besides being the emitting materials, the 4-(2-(4-(diphenylamino)phenyl)-1H-phenanthro[9,10-d]imidazol-1-yl)benzonitrile (TPMCN, Fig. 1) was also used as host material with EQE up to $6.3 \%$ and exciton utilizing efficiency of $64 \%^{22}$. More recently, triphenylamine-acridine (TPA-9AC, Fig. 1) was reported to be a visualization and ultrasensitive fluorescence sensor for diethyl chlorophosphate vapor fast and accurate detection ${ }^{23}$.

Taking the carbazole-dicyanobenzene molecule 4CzIPN (Fig. 1) and triphenylamine-thiadiazole molecule TPA-NZP (Fig. 1) as theoretical models, Pan et al. pointed out different reverse intersystem crossing (RISC) paths $\left(\mathrm{T}_{1} \rightarrow \mathrm{S}_{1}\right.$ for $4 \mathrm{CzIPN}$ and $\mathrm{T}_{2} \rightarrow \mathrm{S}_{2}$ for TPA-NZP) by comparing the singlet exciton utilization formats using density functional theory calculation ${ }^{24}$. The systematic calculations on accurate description of HLCT state also

${ }^{1}$ College of Science, Beihua University, Jilin 132013, People's Republic of China. ${ }^{2}$ Jilin Provincial Key Laboratory of Straw-Based Functional Materials, Institute for Interdisciplinary Biomass Functional Materials Studies, Jilin Engineering Normal University, Changchun 130052, People's Republic of China. ${ }^{3}$ Faculty of Chemistry, Northeast Normal University, Changchun 130024, People's Republic of China. ${ }^{\bowtie}$ email: ybt199@126.com; gaoy029@163.com 


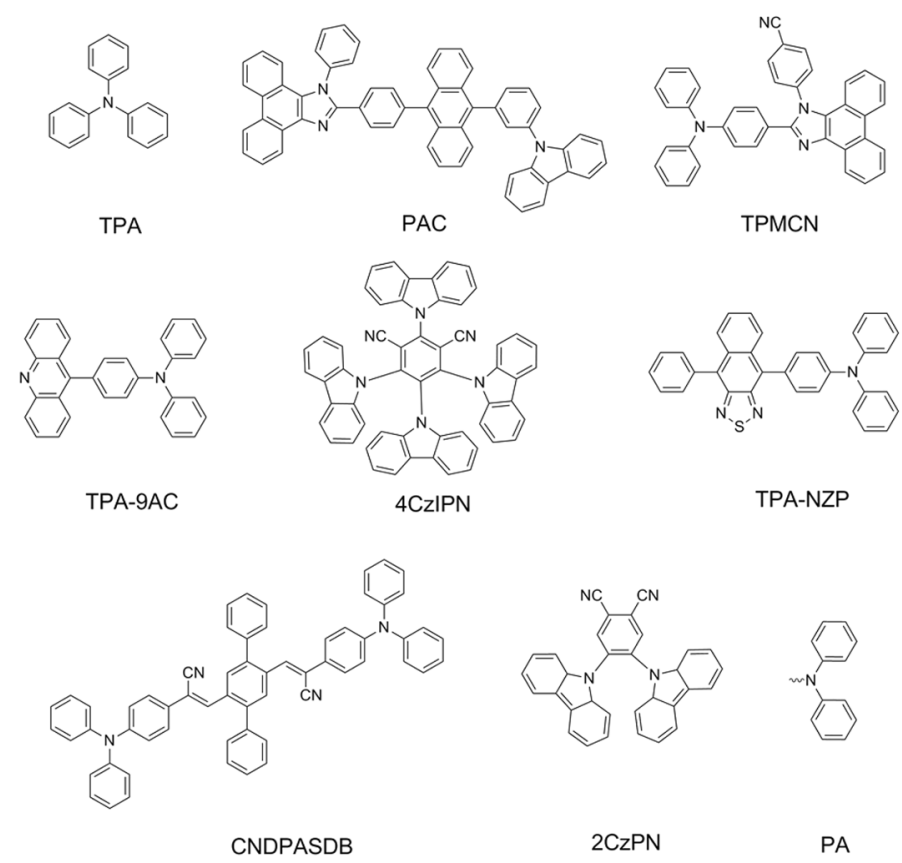

Figure 1. Molecular structures extracted from references.

demonstrate that the $\omega \mathrm{B} 97 \mathrm{XD}$ or optimally tuned range-separated functional can provide a better description of HLCT state st,26. $^{25}$.

It is observed that molecules containing TPA group show a sharp emission wavelength dependence on solvent polarity ${ }^{27-30}$. For example, the work done by Xia et al. proposed the explanation that the emissions of cyano-substituted oligo a-phenylenevinylene-1,4-bis( $R$-cyano-4-diphenylaminostyryl)-2,5-diphenylbenzene (CNDPASDB, Fig. 1) were from the LE state in a low-polarity solvent but were from the CT state in the highpolarity solvent ${ }^{31}$. An overall theoretical investigation of TPA-NZP also concluded the excited state changed from HLCT character to complete intramolecular CT with increasing solvent polarities, followed by increased non-radiative decay rate r $^{32}$

Huang et al. also suggested an aggregation-induced emission mechanism that a conversion is from dark twisted intramolecular charge transfer (TICT) excited state to emissive quasi-TICT state because that the restriction of molecular rotation was lower in the amorphous film than in power and crystalline ${ }^{33}$. The fluorescent molecules atomistic molecular dynamics simulation of deposition process showed that the torsion angles between donor and acceptor groups have a broadened distribution around 90 degrees due to the thermal fluctuation and intermolecular interaction ${ }^{34}$. Based on reported compounds (Fig. 1), namely 1,2-bis(carbazol-9-yl)-4,5-dicyanobenzene $(2 \mathrm{CzPN})$ and $(4 \mathrm{CzIPN})$, the theoretical simulation of bulk amorphous phase also indicated that the torsion angles between donor and acceptor are in a broad distribution, and the RISC is a dynamical process with the varying molecular structures ${ }^{35,36}$.

$\mathrm{Ma}$ et al. have proposed the hot-exciton RISC path of the higher triplet state to the single state $\left(\mathrm{T}_{2} \rightarrow \mathrm{S}_{1} /\right.$ $\mathrm{S}_{2}$ ) to explain the large proportion of radiative singlet exciton for HLCT-state molecules. However, we wonder whether the excited-state $\left(S_{1}, T_{1}\right.$, and $\left.T_{2}\right)$ energy levels would lead to the variations between $S_{1}$ and $T_{2}$ gaps and affect the RISC process with molecular rotation. Thus, in this work, taking reported HLCT-state molecules (1, 2, and 3) as examples (Fig. 2a), we have calculated the excited-state potential energy surface (PES) by twisting TPA (Fig. 1) or diphenylamine (PA, Fig. 1) using the time-dependent density functional theory (TD-DFT) method. The calculated results indicate that twisting TPA or PA almost has no influence on $\mathrm{T}_{1}$ and $\mathrm{T}_{2}$ energy levels, but would affect the $S_{1}$ energy level and the $T_{2} \rightarrow S_{1}$ RISC process. Therefore, understanding the relationship between the $S_{1}$ PES and molecular structures is important for designing high-performance luminescent materials utilizing HLCT state.

\section{Computational details}

In this work, the ground-state $\left(\mathrm{S}_{0}\right)$ geometries of $\mathbf{1}, \mathbf{2}$, and 3 (Fig. 2a) were optimized at density functional theory (DFT) calculations M06-2X/Def2SVP level. The lowest singlet excited state $\left(\mathrm{S}_{1}\right)$ was obtained with timedependent density functional theory (TD-DFT) M06-2X/Def2SVP level. Two low-lying triplet excited-state geometries $\left(\mathrm{T}_{1}\right.$ and $\mathrm{T}_{2}$ ) were optimized using the same M06-2X/Def2SVP considering the Tamm-Dancoff approximation (TDA) ${ }^{37,38}$ for avoiding the issue of triplet instability. The corrected linear response (cLR) approach ${ }^{39}$ was employed to obtain the total energies of $S_{1}, T_{1}$, and $T_{2}$ excited-state structures. In all calculations, the dimethylsulfoxide (DMSO) solvent under the SMD model ${ }^{40}$ was used to consider the solvent effect because that the high polar DMSO was prone to stabilize the charge-transfer state. To analyze the excited-state $\left(\mathrm{S}_{1}, \mathrm{~T}_{1}\right.$, and $\left.\mathrm{T}_{2}\right)$ 
a

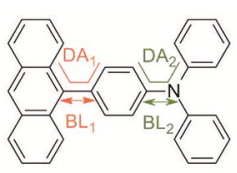

1

b

$\mathrm{b}$

$\mathrm{T}_{2}$

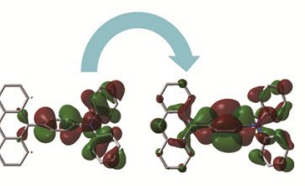

$\mathrm{v}=90 \%$

$\mathrm{T}_{1}$

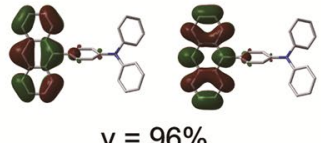

$v=96 \%$

$\mathrm{S}_{1}$

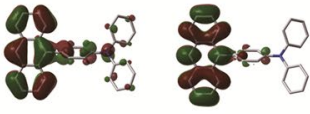

$v=98 \%$

C

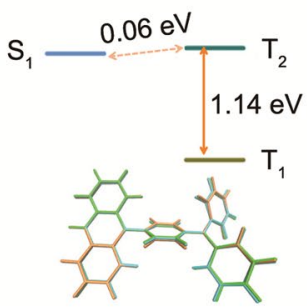

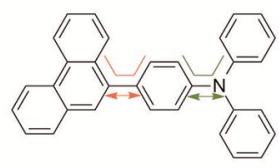

2

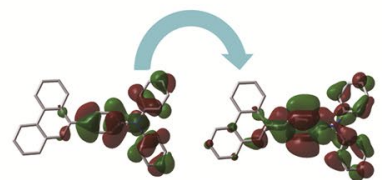

$v=71 \%$

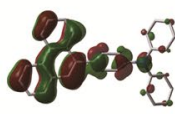

$v=80 \%$

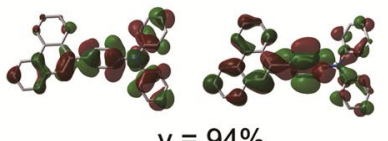

$v=94 \%$

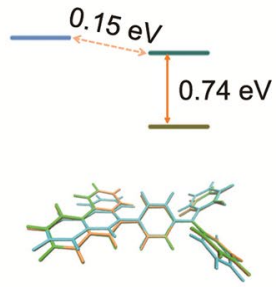

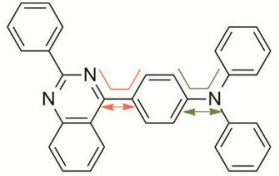

3
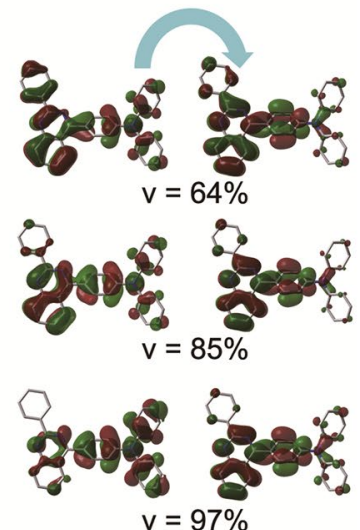

$v=97 \%$

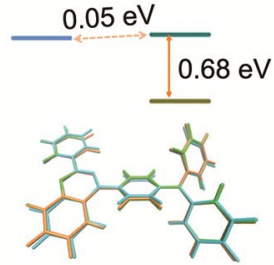

Figure 2. (a) Molecular structures of 1, 2, and 3. (b) Natural transition orbitals of $S_{1}, T_{1}$, and $T_{2}$ excited-state with M06-2X/Def2SVP based on ground-state structures. (c) Relative $S_{1}, T_{1}$, and $T_{2}$ energy levels with M06-2X/ Def2SVP and a structural comparison among $\mathrm{S}_{1}, \mathrm{~T}_{1}$, and $\mathrm{T}_{2}$ structures. $\mathrm{S}_{1}$ (orange); $\mathrm{T}_{1}$ (green); $\mathrm{T}_{2}$ (cyan). (b) Drawn by GaussView 6.0 and Multiwfn programs; (c) is drawn by VMD 1.9.1. program.

properties, the vertical singlet and triplet excited states with M06-2X/Def2SVP were calculated based on their $\mathrm{S}_{0}$ structures. Besides, the $\mathrm{S}_{1}, \mathrm{~T}_{1}$ and $\mathrm{T}_{2}$ excited-state potential energy surface (PES) was performed by twisting TPA or PA group with a scan step of 10 degrees. All calculations were accomplished by Gaussian 16 program $^{41}$. The natural transition orbitals, electron and hole analysis and structural comparison among $\mathrm{S}_{1}, \mathrm{~T}_{1}$, and $\mathrm{T}_{2}$ excited states were obtained by Multiwfn program ${ }^{42}$.

Consent to participate. All authors agree to participate.

Consent for publication. All authors agree for publication.

\section{Result and discussion}

Excited-state properties of 1, 2 and 3. Most reported works explain the high exciton utilization of HLCT-state molecules by analyzing the vertical singlet and triplet excited-state energy levels. In this work, we have optimized the ground-state, singlet, and triplet excited-state structures $\left(\mathrm{S}_{0}, \mathrm{~S}_{1}, \mathrm{~T}_{1}\right.$, and $\left.\mathrm{T}_{2}\right)$ and calculated their relatively adiabatic energies. For better describing the excited-state properties, we also illustrate the natural transition orbitals (NTOs) and the associated weights $(\mathrm{v})$ for the singlet $\left(\mathrm{S}_{1}\right)$ and triplet $\left(\mathrm{T}_{1}\right.$ and $\left.\mathrm{T}_{2}\right)$ states. However, the v values for some excited states are far away from $100 \%$ (in Fig. $2 \mathrm{~b}$ ), and the NOTs are not enough to describe the excited-state properties. Therefore, we further to depict the hole and electron distribution to supplement the NTOs. To analyze the excited states $\left(\mathrm{S}_{1}, \mathrm{~T}_{1}\right.$, and $\left.\mathrm{T}_{2}\right)$ properties, the hole and electron distribution were calculated with three methods (CAM-B3LYP, M06-2X, and $\omega$ B97XD) based on their $\mathrm{S}_{0}$ structures.

The overlap of hole and electron (Sr), separation degrees of hole and electron $(\mathrm{t})$, distance between centroid of hole and electron (D), and overall mean span of hole and electron (H) (in Table S1-S3) were used to describe the quantitative representation of hole and electron distribution, and there are tiny differences in these parameters. However, three methods give similar excited-state characteristics for $\mathrm{S}_{1}, \mathrm{~T}_{1}$, and $\mathrm{T}_{2}$ states. Figure $\mathrm{S} 1$ depicts the electron and hole distribution obtained with CAM-B3LYP, $\omega$ B97XD and M06-2X methods. According to the 
hole and electron distribution, the $S_{1}, T_{1}$ and $T_{2}$ states of $\mathbf{1}, \mathbf{2}$, and 3 display main locally excited (LE) and very slight charge-transfer (CT) natures.

We take the plot (Fig. 2b) with M06-2X as an example to discuss the NTOs. As seen in Fig. 2b, the $\mathrm{S}_{1}$ states of 1, 2, and 3 display main LE and very slight CT natures. For example, the NTOs of 1 mainly locate on the anthracene group and the middle phenyl ring with little CT from the TPA to anthracene group. Both NOTs of $\mathbf{2}$ and $\mathbf{3}$ are delocalized over the whole molecules. Such high LE percentages in $S_{1}$ states were beneficial for high fluorescence radiative rate. Similarly, the $\mathrm{T}_{1}$ states of $\mathbf{1 , 2}$, and $\mathbf{3}$ also possess main LE and little CT natures. For example, the NTOs of $\mathbf{1}$ are mainly distributed on the anthracene group, and both $\mathbf{2}$ and $\mathbf{3}$ display the NTOs over 2-phenylquinazoline group and middle phenyl ring. As for $T_{2}, \mathbf{1}, \mathbf{2}$, and $\mathbf{3}$ have dominant LE characteristics. 1 has NTOs over the TPA group, and the NTOs of $\mathbf{2}$ and $\mathbf{3}$ locate over the whole molecules.

Taking $\mathrm{S}_{0}$ as a reference, the relative $\mathrm{S}_{1}, \mathrm{~T}_{1}$, and $\mathrm{T}_{2}$ energy levels are depicted in Fig. 2c. For all molecules (1, 2 , and 3), the $T_{2}$ is quite close to $S_{1}$ in energy levels, and such a small $T_{2}$ and $S_{1}$ gaps are apt to realize $T_{2} \rightarrow S_{1}$ reverse intersystem crossing (RISC) path. Besides, compared to the $S_{1}$ and $T_{2}$ gaps, the $T_{2}$ and $T_{1}$ gaps of all molecules $\left(1,2\right.$, and 3 ) are relatively large and beneficial to suppress the $T_{1}$ and $T_{2}$ internal conversion, leading to more $T_{2}$ population. The combination of more $T_{2}$ population and negligible $T_{2}$ and $S_{1}$ gaps is advantage for $\mathrm{T}_{2} \rightarrow \mathrm{S}_{1}$ RISC, which is consistent with the explanation of the large proportion of radiation singlet exciton for HLCT-state molecules. The $\mathbf{1}$ and $\mathbf{2}$ have similar structures, but the $\mathrm{T}_{1}$ and $\mathrm{T}_{2}$ gap of $\mathbf{1}$ and $\mathbf{2}$ are $1.14 \mathrm{eV}$ and $0.75 \mathrm{eV}$, indicating a slight geometric variation has a huge influence on $\mathrm{T}_{1}$ and $\mathrm{T}_{2}$ energy gap. Consequently, a careful group combination for designing novel luminescent materials utilizing HLCT state is important.

To quantify the geometric change, we also summarize the typical bond lengths (BL1 and BL2) and dihedral angles (DA1 and DA2) (shown in Fig. $2 \mathrm{a}$ and Table $\mathrm{S} 4$ ) in $\mathrm{S}_{0}, \mathrm{~S}_{1}, \mathrm{~T}_{1}$, and $\mathrm{T}_{2}$ structures. Upon electron excitation from $S_{0}$ to $S_{1}, T_{1}$ and $T_{2}$, the DA1 have more noticeable variation $\left(-45.9\right.$ to $-69.0^{\circ}$ for 1$)$ than DA2 $\left(28.8^{\circ}-38.9^{\circ}\right.$ for 1). Likewise, the BL2 variation between $S_{0}$ and excited state $\left(S_{1}, T_{1}\right.$, and $\left.T_{2}\right)$ is smaller than BL1, and the differences are below $0.03 \AA$. The BL1 and BL2 variations show the opposite variations to DA1 and DA2. The larger rotations lead to shorter bond lengths between TPA (or PA) and various groups. Figure $2 \mathrm{c}$ depicts a geometric comparison among the $\mathrm{S}_{1}, \mathrm{~T}_{1}$, and $\mathrm{T}_{2}$, and the structure deviation are mostly originated from the DA1 and DA2.

Singlet excited-state potential energy surface by twisting TPA or PA group. The results with non-restricted optimized $\mathrm{S}_{1}, \mathrm{~T}_{1}$, and $\mathrm{T}_{2}$ structures are consistent with the hot-exciton RISC path proposed by Ma et al. However, upon the excited states $\left(\mathrm{S}_{1}, \mathrm{~T}_{1}\right.$, and $\left.\mathrm{T}_{2}\right)$, the stable CT state formation by twisting TPA or PA would affect the $T_{2}$ and $S_{1}$ gap and RISC process. On one hand, if the CT state is the most stable emission state, the decrease of the $S_{1}$ state would affect the quantum efficiency by decreasing the fluorescence decay rate and aggravating the non-radiative relaxation. On the other hand, the enlarging $S_{1}$ and $T_{2}$ gap originated from the decreased $S_{1}$ energy level would weaken the $T_{2}$ exciton utilization. Like-TADF molecules based on donor and acceptor groups through a single bond, the molecular rotation was easily activated and would generate a large spectrum of conformations at room temperature ${ }^{43}$. For 1, 2, and 3 (Fig. 2a), the donor and acceptor groups are connected via a flexible single bond, therefore, the $\mathrm{S}_{1}, \mathrm{~T}_{1}$, and $\mathrm{T}_{2} \mathrm{CT}$ states by twisting TPA or PA were calculated.

At first, we performed the triplet excited-state $\left(\mathrm{T}_{1}\right.$ and $\left.\mathrm{T}_{2}\right)$ potential energy surface (PES) (in Figure S2) with twisting TPA or PA. It can be found that almost all $\mathrm{T}_{1}$ and $\mathrm{T}_{2}$ energies are increased with twisting TPA or PA, meaning that molecular rotations are difficult to realize upon $\mathrm{T}_{1}$ and $\mathrm{T}_{2} \mathrm{PES}$. Consequently, the $\mathrm{T}_{1}$ and $\mathrm{T}_{2}$ energy levels are almost constant. Further, the singlet excited-state potential energy surface $\left(\mathrm{S}_{1} \mathrm{PES}\right)$ with twisting TPA or PA with a scan step of 10 degrees has been performed.

The vertical $\mathrm{S}_{1}$ states of three HLCT-state molecules based on $\mathrm{S}_{0}$ structures (in Table S5) display large oscillator strength in a range from 0.3560 to 0.9085 , indicating main LE transition and high fluorescence emission. Figure $3 \mathrm{~b}$ depicts the highest occupied molecular orbital (HOMO) and lowest unoccupied molecular orbital $(\mathrm{LUMO})$. All $\mathrm{S}_{1}$ states are attributed to a mixture of more HOMO $\rightarrow$ LUMO and less HOMO-1 $\rightarrow$ LUMO contributions (Fig. 3a). For example, 3 possesses a total HOMO $\rightarrow$ LUMO (80\%) transition with HOMO mainly diffused over TPA and LUMO localized on the 2-phenylquinazoline group (Fig. 3b). The HOMO-1 of $\mathbf{1}$ is distributed over the whole molecules, and the HOMO- $1 \rightarrow$ LUMO transition presents the main LE characteristic. The $\mathrm{S}_{1}$ states of $\mathbf{1}, \mathbf{2}$, and 3 molecules display a combination of CT and LE features.

In terms of twisting TPA $\left(\mathrm{DA}_{1}\right)$ or PA $\left(\mathrm{DA}_{2}\right)($ Fig. $3 \mathrm{c}, \mathrm{d})$, the plots of $\mathrm{S}_{1}$ PES show three variation tendencies. It can be concluded that the CT state of $\mathbf{3}$ is very easy to realize by twisting TPA or PA. But the TPA or PA of 2 is very different to twist on the $\mathrm{S}_{1}$ PES. The CT state formation of $\mathbf{1}$ is in a barrierless process by twisting TPA, but not for PA. To illustrate the excited-state properties, Table S6-S13 lists the oscillator strength, transition configuration, and frontier molecular orbitals of 1, 2, and $\mathbf{3}$ with twisting TPA or PA.

On the $S_{1}$ PES of $\mathbf{1}$ and $\mathbf{3}$ with twisting TPA, the CT state is more stable than the HLCT state and would become the finally stable emission state, suppressing small energy barriers $(0.05 \mathrm{eV}$ for $\mathbf{1}$ and $0.02 \mathrm{eV}$ for $\mathbf{3})$. At small $\mathrm{DA}_{1}$, the $\mathrm{S}_{1}$ states of $\mathbf{1}$ contain a main $\mathrm{HOMO} \rightarrow$ LUMO transition, but a mixture of $\mathrm{HOMO} \rightarrow$ LUMO and HOMO- $\rightarrow$ LUMO transitions at $70^{\circ}-80^{\circ}$ is found. With increasing $\mathrm{DA}_{1}$, the HOMO shifts from the whole molecules to the TPA group, and LUMO moves from the anthracene and the middle phenyl ring to the whole anthracene (Table S8). Finally, the $\mathrm{S}_{1}$ state $\left(\mathrm{DA}_{1}=90\right.$ degrees) is a total CT character with a low $\mathrm{f}$ (nearly zero). The $S_{1}$ states of 3 are assigned to $\mathrm{HOMO} \rightarrow$ LUMO in a percentage range of $20 \% \rightarrow 90 \%$ with increased $\mathrm{DA}_{1}$. Considering orbital distribution (Table S10), the initial HOMO shows a high degree of localization of electron

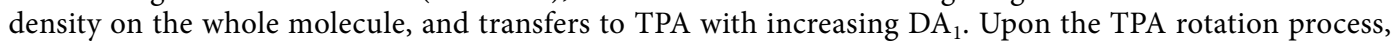
LUMO is always distributed on the 2-phenylquinazoline and the middle phenyl ring. When $\mathrm{DA}_{1}$ is 90 degrees, a noticeably separated HOMO and LUMO for CT state is formed, and $\mathrm{f}$ is nearly approaching to zero. However, the $S_{1}$ energies of 2 are increasing with twisting TPA (Fig. 3c). The energy is the highest point on the $S_{1}$ PES $\left(\mathrm{DA}_{1}=90\right.$ degrees $)$, indicating that TPA rotation of $\mathbf{2}$ is restrained. Therefore, the relative $S_{1}$ and $T_{1}$ energy levels 
a

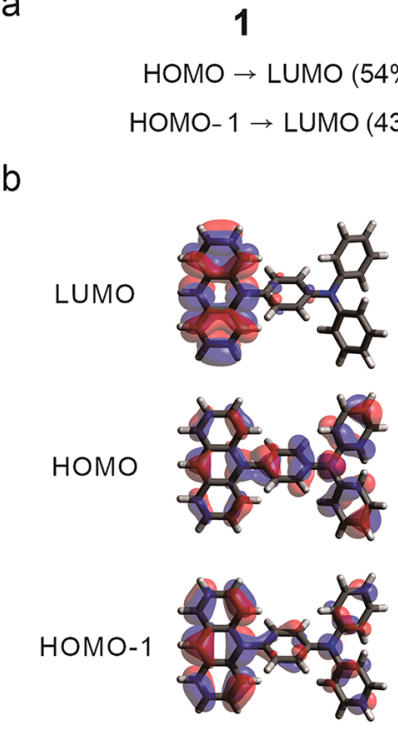

C

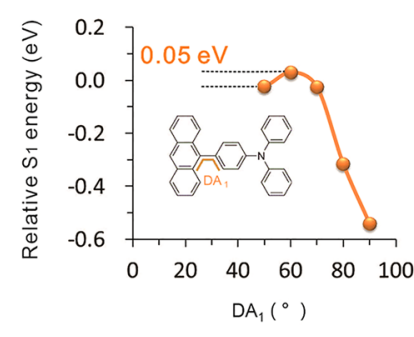

d

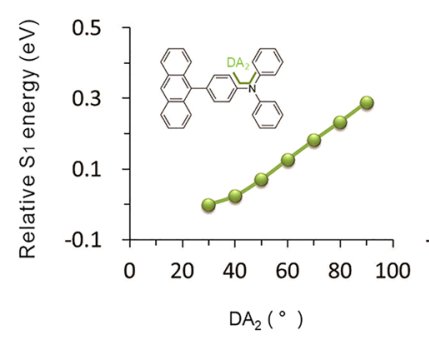

2

HOMO $\rightarrow$ LUMO $(68 \%)$
3

HOMO $\rightarrow$ LUMO $(80 \%)$
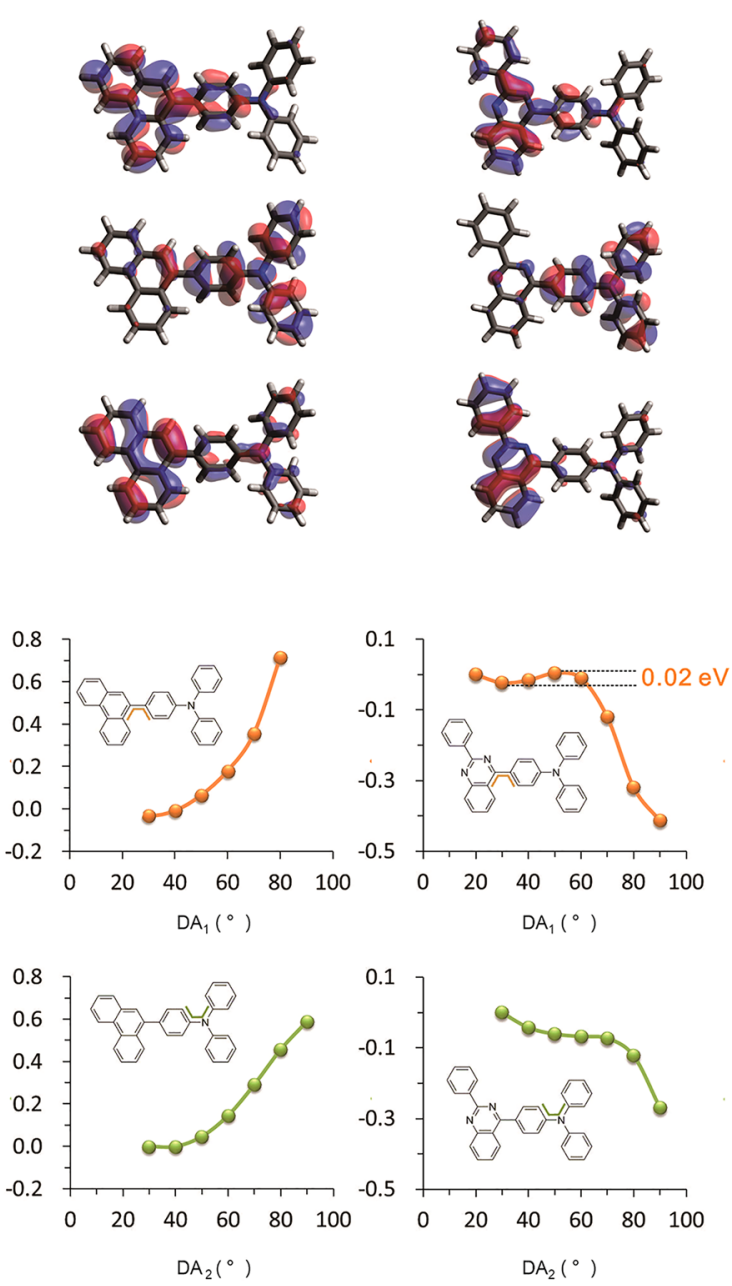

Figure 3. (a) $\mathrm{S}_{1}$ transition configuration of $\mathbf{1}, \mathbf{2}$, and $\mathbf{3}$, (b) HOMO-1, HOMO and LUMO distribution, (c) $\mathrm{S}_{1}$ PES by twisting TPA, (d) $S_{1}$ PES by twisting PA.

are almost unchanged and have no influence on the $\mathrm{T}_{2} \rightarrow \mathrm{S}_{1}$ RISC process. Different from other HLCT molecules, the $\mathrm{S}_{1}$ states have large $\mathrm{f} \mathrm{values}$ in a range from 0.991 to 1.433 (Table S6). With twisting TPA group, the transition configurations (Table S9) change from HLCT to total LE, accompanied by high $\mathrm{f}$.

As with twisting PA (Fig. 3d), the relative energies of $\mathbf{1}$ and $\mathbf{2}$ increase linearly with increasing $\mathrm{DA}_{2}$, indicating that the PA rotation of $\mathbf{1}$ and $\mathbf{2}$ on $\mathrm{S}_{1}$ PES need to overcome the high energy barrier to realize. The $\mathrm{S}_{1}$ states of $\mathbf{1}$ contain a major HOMO $\rightarrow$ LUMO (Table S7). As with twisting PA, the HOMO is initially delocalized over the whole molecules and shifts to the anthracene and middle phenyl ring, and the LUMO show almost complete localization on the anthracene and middle phenyl ring. Finally, the $\mathrm{S}_{1}$ state $\left(\mathrm{DA}_{2}=90\right.$ degrees $)$ of 1 contains the main LE state and has non-zero $\mathrm{f}$ (Table $\mathrm{S} 6$ ). The $\mathrm{S}_{1}$ states of $\mathbf{2}$ contain a major $\mathrm{HOMO} \rightarrow \mathrm{LUMO}$ and minor HOMO- $1 \rightarrow$ LUMO. Along twisting PA, the HOMO and HOMO- 1 are always localized over the whole molecules, and LUMO is distributed on the phenanthrene and middle phenyl ring (Table S12). When the $\mathrm{DA}_{2}$ is 90 degrees, the $S_{1}$ state displays the main LE characteristic and large $f\left(\right.$ Table S6). With increasing $D_{2}$, the $S_{1}$ energy of 3 is decreasing, and a stable $\mathrm{CT}$ state would form in a barrierless process by twisting PA. The HOMO $\rightarrow$ LUMO $(84-90 \%)$ transition gives dominant contributions to $\mathrm{S}_{1}$ states along twisting PA. At small $\mathrm{DA}_{2}$, the $\mathrm{HOMO}$ is mostly distributed on the TPA and a small part on the 2-phenylquinazoline but shift to the TPA group with increasing $\mathrm{DA}_{2}$ (Table S13). When the $\mathrm{DA}_{2}$ is 90 degrees, the HOMO is centered on the PA group, while LUMO are distributed on 2-phenylquinazoline and middle phenyl ring with $\mathrm{f}$ nearly to zero for $\mathrm{S}_{1}$ state.

In the excited state, the CT states $\left(\mathrm{DA}_{1}=90\right.$ degrees $)$ of $\mathbf{1}$ and $\mathbf{3}\left(\mathrm{DA}_{1} / \mathrm{DA}_{2}=90\right.$ degrees $)$ are easy to populate via twisting TPA or PA, accomplished by decreased $\mathrm{f}$ and $\mathrm{S}_{1}$ emission efficiency. Moreover, the formation of stable CT states would enlarge the $S_{1}$ and $T_{2}$ gap, weaken the $T_{2}$ to $S_{1}$ RISC and decrease the triplet exciton utilization. 


\section{Conclusion}

In conclusion, taking molecules $\mathbf{1}, \mathbf{2}$, and $\mathbf{3}$ as examples, we have investigated the excited-state potential energy surface (PES) by twisting triphenylamine (TPA) or diphenylamine (PA) by density functional theory and timedependent density functional theory calculations. The calculated relative total energy indicated that the $\mathrm{T}_{2}$ and $T_{1}$ energy gap is large, and $T_{2}$ is closed to $S_{1}$ in energy levels under the case of non-restricted excited-state optimizations. Meanwhile, the $S_{1}$ states of 1,2 , and 3 have a mixture of locally excited (LE) and charge-transfer (CT) characteristics, high oscillator strength, and high fluorescence emission efficiency. Considering the excited state PES, the $T_{1}$ and $T_{2}$ energy levels are almost constant. However, for the $S_{1} P E S$, it can be found that twisting TPA or PA would stabilize the CT state, which would seriously damage the quantum efficiency. For example, the CT states of $\mathbf{3}$ are very easy to realize no matter by twisting TPA or PA. The stable CT state formation of $\mathbf{3}$ would enlarge the energy gap between $S_{1}$ and $T_{2}$ and decrease the $T_{2}$ exciton utilization. However, the TPA or PA group of 2 is very different to twist in the $S_{1}$ PES, and $S_{1}$ and $T_{2}$ energy gap is almost unchanged. For $\mathbf{1}$, the CT states are formed in a barrierless process by twisting TPA, but not for PA. Therefore, understanding the relationship between the $S_{1}$ PES and molecular structures is important for designing high-performance luminescent materials utilizing HLCT state.

\section{Data availability}

The authors declare that the data and manuscript are availability.

Received: 4 May 2021; Accepted: 23 August 2021

Published online: 03 September 2021

\section{References}

1. Tang, C. W. \& VanSlyke, S. A. Organic electroluminescent diodes. Appl. Phys. Lett. 51, 913-915. https://doi.org/10.1063/1.98799 (1987).

2. Baldo, M. A. et al. Highly efficient phosphorescent emission from organic electroluminescent devices. Nature 395, 151-154. https:// doi.org/10.1038/25954 (1998).

3. Baldo, M. A., Lamansky, S., Burrows, P. E., Thompson, M. E. \& Forrest, S. R. Very high-efficiency green organic light-emitting devices based on electrophosphorescence. Appl. Phys. Lett. 75, 4-6. https://doi.org/10.1063/1.124258 (1999).

4. Chen, X. K., Kim, D. \& Brédas, J. L. Thermally activated delayed fluorescence (TADF) path toward efficient electroluminescence in purely organic materials: Molecular level insight. Acc. Chem. Res. 51, 2215-2224. https://doi.org/10.1021/acs.accounts.8b001 74 (2018).

5. Uoyama, H., Goushi, K., Shizu, K., Nomura, H. \& Adachi, C. Highly efficient organic light-emitting diodes from delayed fluorescence. Nature 492, 234-238. https://doi.org/10.1038/nature11687 (2012).

6. Yang, Z. Y. et al. Recent advances in organic thermally activated delayed fluorescence materials. Chem. Soc. Rev. 46, 915-1016. https://doi.org/10.1039/C6CS00368K (2017).

7. Li, W. J. et al. A twisting donor-acceptor molecule with an intercrossed excited state for highly efficient, deep-blue electroluminescence. Adv. Funct. Mater. 22, 2797-2803. https://doi.org/10.1002/adfm.201200116 (2012).

8. Li, W. J. et al. Employing $\sim 100 \%$ excitons in OLEDs by utilizing a fluorescent molecule with hybridized local and charge-transfer excited state. Adv. Funct. Mater. 24, 1609-1614. https://doi.org/10.1002/adfm.201301750 (2014).

9. Han, X. et al. Highly efficient solid-state near-infrared emitting material based on triphenylamine and diphenylfumaronitrile with an EQE of $2.58 \%$ in nondoped organic light-emitting diode. Adv. Funct. Mater. 25, 7521-7529. https://doi.org/10.1002/adfm.20150 3344 (2015).

10. Li, B. J. et al. Dual-emissive 2-(2'-hydroxyphenyl)oxazoles for high performance organic electroluminescent devices: Discovery of a new equilibrium of excited state intramolecular proton transfer with a reverse intersystem crossing process. Chem. Sci. 9, 1213-1220. https://doi.org/10.1039/C7SC04464J (2018).

11. Zhou, C. J. et al. Enhancing the electroluminescent efficiency of acridine-based donor-acceptor materials: Quasi-equivalent hybridized local and charge-transfer state. J. Phys. Chem. C 122, 18376-18382. https://doi.org/10.1021/acs.jpcc.8b07083 (2018).

12. Liu, B. et al. Ambipolar D-A type bifunctional materials with hybridized local and charge-transfer excited state for high performance electroluminescence with EQE of 7.20\% and CIEy 0.06. J. Mater. Chem. C 5, 5402-5410. https://doi.org/10.1039/C7TC0 1133D (2017).

13. Li, C. et al. Effect of cyano-substitution in distyrylbenzene derivatives on their fluorescence and electroluminescence properties. J. Mater. Chem. C 4, 7478-7484. https://doi.org/10.1039/C6TC01886F (2016).

14. Konidena, R. K., Thomas, K. R. J., Dubey, D. K., Sahoo, S. \& Jou, J. H. A new molecular design based on hybridized local and charge transfer fluorescence for highly efficient (>6\%) deep-blue organic light emitting diodes. Chem. Commun. 53, 11802-11805. https:// doi.org/10.1039/c7cc07139f (2017).

15. Yang, B. et al. The origin of the improved efficiency and stability of triphenylamine-substituted anthracene derivatives for OLEDs: A theoretical investigation. ChemPhysChem 9, 2601-2609. https://doi.org/10.1002/cphc.200800513 (2008).

16. Li, W. J. et al. A hybridized local and charge-transfer excited state for highly efficient fluorescent OLEDs: Molecular design, spectral character, and full exciton utilization. Adv. Opt. Mater. 2, 892-901. https://doi.org/10.1002/adom.201400154 (2014).

17. Zhang, S. T. et al. Enhanced proportion of radiative excitons in non-doped electro-fluorescence generated from an imidazole derivative with an orthogonal donor-acceptor structure. Chem. Commun. 49, 11302-11304. https://doi.org/10.1039/C3CC47130F (2013).

18. Yao, L. et al. Highly efficient near-infrared organic light-emitting diode based on a butterfly-shaped donor-acceptor chromophore with strong solid-state fluorescence and a large proportion of radiative excitons. Angew. Chem. Int. Ed. 53, 2119-2123. https://doi. org/10.1002/anie.201308486 (2014).

19. Zhang, S. T. et al. Achieving a significantly increased efficiency in nondoped pure blue fluorescent OLED: A quasi-equivalent hybridized excited state. Adv. Funct. Mater. 25, 1755-1762. https://doi.org/10.1002/adfm.201404260 (2015).

20. Liu, H. C. et al. Highly efficient near ultraviolet organic light-emitting diode based on a meta-linked donor-acceptor molecule. Chem. Sci. 6, 3797-3804. https://doi.org/10.1039/C5SC01131K (2015).

21. Xu, Y. W. et al. Highly efficient blue fluorescent OLEDs based on upper level triplet-singlet intersystem crossing. Adv. Mater. 31, 1807388. https://doi.org/10.1002/adma.201807388 (2019).

22. Chen, L. F. et al. Breaking the efficiency limit of fluorescent OLEDs by hybridized local and charge-transfer host materials. J. Phys. Chem. Lett. 9, 5240-5245. https://doi.org/10.1021/acs.jpclett.8b02138 (2018). 
23. Li, X. B. et al. Visualization of ultrasensitive and recyclable dual-channel fluorescence sensors for chemical warfare agents based on the state dehybridization of hybrid locally excited and charge transfer materials. Anal. Chem. 91, 10927-10931. https://doi.org/ 10.1021/acs.analchem.9b02085 (2019).

24. Pan, Y. Y. et al. High yields of singlet excitons in organic electroluminescence through two paths of cold and hot excitons. Adv. Opt. Mater. 2, 510-515. https://doi.org/10.1002/adom.201300467 (2014).

25. Pan, Y. Y. et al. Accurate description of hybridized local and charge-transfer excited-state in donor-acceptor molecules using density functional theory. RSC Adv. 6, 108404-108410. https://doi.org/10.1039/C6RA22267F (2016).

26. Jiang, Y. R. et al. Accurate prediction for dynamic hybrid local and charge transfer excited states from optimally tuned rangeseparated density functionals. J. Phys. Chem. C 123, 5616-5625. https://doi.org/10.1021/acs.jpcc.9b00027 (2019).

27. Kautny, P. et al. Charge-transfer states in triazole linked donor-acceptor materials: Strong effects of chemical modification and solvation. Phys. Chem. Chem. Phys. 19, 18055-18067. https://doi.org/10.1039/C7CP01664F (2017).

28. Zhou, C. J. et al. Investigation on excited-state properties and electroluminescence performance of donor-acceptor materials based on quinoxaline derivatives. Org. Electron. 75, 105414. https://doi.org/10.1016/j.orgel.2019.105414 (2019).

29. Li, Y. X., Tan, T. F., Wang, S. T., Xiao, Y. \& Li, X. G. Highly solvatochromic fluorescence of anthraquinone dyes based on triphenylamines. Dyes Pigments 144, 262-270. https://doi.org/10.1016/j.dyepig.2017.05.024 (2017).

30. Shen, Y. et al. Enhanced deep-red emission in donor-acceptor molecular architecture: The role of ancillary acceptor of cyanophenyl. Chin. Chem. Lett. 30, 1947-1950. https://doi.org/10.1016/j.cclet.2019.07.059 (2019).

31. Song, H. W. et al. Solvent modulated excited state processes of push-pull molecule with hybridized local excitation and intramolecular charge transfer character. Phys. Chem. Chem. Phys. 21, 3894-3902. https://doi.org/10.1039/C8CP06459H (2019).

32. Fan, D. et al. Solvent effects on the optical spectra and excited-state decay of triphenylamine-thiadiazole with hybridized local excitation and intramolecular charge transfer. J. Phys. Chem. A 119, 5233-5240. https://doi.org/10.1021/jp5099409 (2015).

33. Li, J. W. et al. From dark TICT state to emissive quasi-TICT state: The AIE mechanism of N-(3-(benzo[d]oxazol-2-yl)phenyl)4-tert-butylbenzamide. J. Phys. Chem. C 119, 2133-2141. https://doi.org/10.1021/jp5089433 (2015).

34. Hu, T. P., Han, G. C., Tu, Z. Y., Duan, R. H. \& Yi, Y. P. Origin of high efficiencies for thermally activated delayed fluorescence organic light-emitting diodes: Atomistic insight into molecular orientation and torsional disorder. J. Phys. Chem. C 122, $27191-27197$. https://doi.org/10.1021/acs.jpcc.8b08169 (2018).

35. Moral, M., Son, W. J., Sancho-García, J. C., Oivier, Y. \& Muccioli, L. Cost-effective force field tailored for solid-phase simulations of OLED materials. J. Chem. Theory Comput. 11, 3383-3392. https://doi.org/10.1021/acs.jctc.5b00164 (2015).

36. Olivier, Y. et al. Nature of the singlet and triplet excitations mediating thermally activated delayed fluorescence. Phys. Rev. Mater. 1, 075602. https://doi.org/10.1103/PhysRevMaterials.1.075602 (2017).

37. Peach, M. J. G., Williamson, M. J. \& Tozer, D. J. Influence of triplet instabilities in TDDFT. J. Chem. Theory Comput. 7, 3578-3585. https://doi.org/10.1021/ct200651r (2011).

38. Hirata, S. \& Head-Gordon, M. Time-dependent density functional theory within the Tamm-Dancoff approximation. Chem. Phys. Lett. 314, 291-299. https://doi.org/10.1016/S0009-2614(99)01149-5 (1999).

39. Caricato, M. et al. Formation and relaxation of excited states in solution: A new time dependent polarizable continuum model based on time dependent density functional theory. J. Chem. Phys. 124, 124520. https://doi.org/10.1063/1.2183309 (2006).

40. Marenich, A. V., Cramer, C. J. \& Truhlar, D. G. Universal solvation model based on solute electron density and on a continuum model of the solvent defined by the bulk dielectric constant and atomic surface tensions. J. Phys. Chem. B 113, 6378-6396. https:// doi.org/10.1021/jp810292n (2009).

41. Frisch, M. J. et al. Gaussian 16, Revision A.03 (Gaussian Inc., 2016).

42. Lu, T. \& Chen, F. W. Multiwfn: A multifunctional wavefunction analyzer. J. Comput. Chem. 33, 580-592. https://doi.org/10.1002/ jcc.22885 (2012).

43. Olivier, Y., Sancho-Garcia, J. C., Muccioli, L., D’Avino, G. \& Beljonne, D. Computational design of thermally activated delayed fluorescence materials: The challenges ahead. J. Phys. Chem. Lett. 9, 6149-6163. https://doi.org/10.1021/acs.jpclett.8b02327 (2018).

\section{Author contributions}

G.S. (0.2), X.-H.W. (0.05), J.L. (0.05), B.-T.Y. (0.3), Y.G. (0.3), Y.G. (0.1). Y.G. suggest the idea of this manuscript; G.S. and B.-T.Y. provide the calculation and writing of the manuscript; X.-H.W., J.L. and Y.G. are responsible for revise of date, figures, tables and text.

\section{Funding}

The authors gratefully acknowledge financial support from the National Natural Science Foundation of China (NSFC) (Grant No. 51902124, 21802002, 51875249), Central Government to Guide Local Science and Technology Development Funds Jilin Province Basic Research Project (Grant No. 202002002JC), Joint Funds of Jilin Natural Science Foundation, Free Exploration Project, Central Government to Guide Local Science and Technology Development Funds, (Grant No. 2020122370JC).

\section{Competing interests}

The authors declare no competing interests.

\section{Additional information}

Supplementary Information The online version contains supplementary material available at https://doi.org/ 10.1038/s41598-021-97229-z.

Correspondence and requests for materials should be addressed to B.-T.Y. or Y.G.

Reprints and permissions information is available at www.nature.com/reprints.

Publisher's note Springer Nature remains neutral with regard to jurisdictional claims in published maps and institutional affiliations. 
(c) (i) Open Access This article is licensed under a Creative Commons Attribution 4.0 International cc) License, which permits use, sharing, adaptation, distribution and reproduction in any medium or format, as long as you give appropriate credit to the original author(s) and the source, provide a link to the Creative Commons licence, and indicate if changes were made. The images or other third party material in this article are included in the article's Creative Commons licence, unless indicated otherwise in a credit line to the material. If material is not included in the article's Creative Commons licence and your intended use is not permitted by statutory regulation or exceeds the permitted use, you will need to obtain permission directly from the copyright holder. To view a copy of this licence, visit http://creativecommons.org/licenses/by/4.0/.

(C) The Author(s) 2021 